\title{
The effect of lipocalin-2 (LCN2) on apoptosis: a proteomics analysis study in an LCN2 deficient mouse model
}

\author{
Dongming Wu, Xiaopeng Wang, Ye Han and Yayun Wang ${ }^{*}$
}

\begin{abstract}
Background: Recent studies have shown that lipocalin-2 (LCN2) has multiple functions involved in various biological and pathological processes including energy homeostasis, cancer, inflammation, and apoptosis. We aimed to investigate the effect of LCN2 on apoptosis that influences the pathogenetic process of metabolic diseases and cancer.

Methods: We performed a proteomics analysis of livers taken from LCN2-knockout mice and wild type mice by using label-free LC-MS/MS quantitative proteomics.

Results: Proteomic analysis revealed that there were 132 significantly differentially expressed proteins (49 upregulated and 83 downregulated) among 2140 proteins in the liver of LCN2-knockout mice compared with wild type mice. Of these, seven apoptosis-associated proteins were significantly upregulated and seven apoptosis-associated proteins downregulated.

Conclusion: Proteomics demonstrated that there were seven upregulated and seven downregulated apoptosisassociated proteins in liver of LCN2-knockout mice. It is important to clarify the effect of LCN2 on apoptosis that might contribute to the pathogenesis of insulin resistance, cancer, and various nervous system diseases.
\end{abstract}

Keywords: Lipocalin 2, Label-free proteomics, Apoptosis

\section{Introduction}

Lipocalin-2 (LCN2), also known as neutrophil gelatinaseassociated lipocalin, is initially an iron-trafficking protein involved in iron metabolism, innate immune function, and antibacterial infection [1-5]. LCN2 is a secreted glycoprotein belonging to the lipocalin superfamily and widely expressed in the bone marrow, gall bladder, colon, urinary bladder, stomach, and liver [1, 6-8]. Human LCN2 protein encoded by $L C N 2$ gene is a $23 \mathrm{kDa}$ protein and has two isoforms by alternative splicing leading to a six amino acid difference in the $\mathrm{C}$-terminus of the protein sequence [9].

\footnotetext{
*Correspondence: wyy13969710295@163.com
}

Department of Emergency, Qingdao Hospital of Traditional Chinese Medicine (Qingdao Hiser Hospital), No. 4, Renmin Road, Shibei District, Qingdao 266033, Shandong Province, China
Recently, accumulating evidence has demonstrated that the LCN2 protein is implicated in a wide range of cellular and pathophysiological processes such as insulin insistence, tumor cell proliferation, and apoptosis [8, 10-14]. Serum LCN2 protein levels were significantly elevated in patients with type 2 diabetes or impaired glucose tolerance as well as in obese women [13, 15]. Furthermore, LCN2-knockout (LCN2-KO) mice represent a metabolic phenotype of reduced fasting glucose, lipid levels, and improved insulin sensitivity. This suggests that LCN2 plays an important role in metabolic disease [16].

LCN2 expression is upregulated in many kinds of cancers and is likely involved in multiple tumorigenic processes including tumor initiation, progression, and metastasis [12]. The upregulated LCN2 protein expression is positively associated with cancer cell migration 
and progression in hepatocellular cancer through the hepatocyte activating growth factor receptor/focal adhesion kinase (FAK) signaling pathway [17].

LCN2 can modulate the apoptotic process by interacting with interleukin-3 (IL-3) and IL-8 to influence tumor progression, metastasis, and prognosis in FL5.12 cells, endometrial cancer, and liver cancer $[4,18,19]$. The LCN2 expression was upregulated in injured gastric mucosa with obese patients. Interestingly, the upregulated LCN2 can exert a beneficial influence on gastric mucosa via inhibition of apoptosis and inflammatory response [8]. Moreover, LCN2-KO using CRISPR/Cas 9 gene editing technology decreases tumor cell proliferation and migration, thereby enhancing cisplatin-induced apoptosis in the human prostate cancer cell line [20].

Apoptosis is known to influence the pathogenetic process of metabolic diseases and cancer. Thus far, little is known about the underlying mechanisms of LCN2 modulation of apoptosis. To investigate the regulatory mechanism of LCN2 on apoptosis, we performed a proteomic analysis of livers taken from LCN2-KO mice and wild type mice by using label-free liquid chromatography with tandem mass spectrometry (LC-MS/MS) quantitative proteomics. The results revealed multiple apoptosis-associated proteins that were upregulated and downregulated, and these might provide new insights for further therapeutic targets for metabolic diseases and cancer.

\section{Materials and methods \\ Animals}

The LCN2-KO mouse model was established as previously described [21]. Five male LCN2-KO mice and five age-matched C57BL6/J wild type (WT) mice were housed in an SPF room of constant humidity (55\%) and temperature $\left(22 \pm 2^{\circ} \mathrm{C}\right)$ under a 12 -h light-dark cycle. The 8 -week-old LCN2-KO mice and WT mice were allowed ad libitum access to food and water for 6 weeks. All mice were euthanized, and their livers were rapidly excised and stored in liquid nitrogen for subsequent analysis.

The study was reviewed and approved by the Animal Care and Use Committee of Qingdao Hospital of Traditional Chinese Medicine (Qingdao, China). All the surgical procedures were performed under sodium pentobarbital anesthesia and designed to minimize suffering.

\section{Sample preparation}

SDT buffer (4\% SDS, $100 \mathrm{mM}$ Tris- $\mathrm{HCl}, \mathrm{pH} 7.6$ ) was added to the lysed liver, and transferred to $2-\mathrm{mL}$ tubes. The lysate was homogenized by an MP Fastprep-24 Automated Homogenizer $(6.0 \mathrm{M} / \mathrm{S}, 30 \mathrm{~s}$, twice) (MP Biomedicals Inc., USA). The homogenate was sonicated and boiled for $15 \mathrm{~min}$. After centrifugation at $14,000 \mathrm{~g}$ for $40 \mathrm{~min}$, the supernatant was filtered using a $0.22-\mu \mathrm{m}$ filter. The filtrate was quantified using the BCA Protein Assay Kit (P0012, Beyotime) [22].

\section{SDS-PAGE separation}

Briefly, $20 \mu$ g protein for each sample was mixed with $6 \times$ loading buffer and boiled for $5 \mathrm{~min}$. The proteins were separated on $12.5 \%$ SDS-PAGE. The protein bands were visualized by Coomassie Blue R-250 staining [23].

\section{Filter-aided Sample Preparation (FASP digestion)}

Briefly, $200 \mu \mathrm{g}$ protein were reduced with $50 \mathrm{mM}$ DTT for $30 \mathrm{~min}$ at $56^{\circ} \mathrm{C}$. Then, the detergent, DTT, and other lowmolecular-weight components were removed using UA buffer (8M Urea, $150 \mathrm{mM}$ Tris- $\mathrm{HCl} \mathrm{pH} 8.5)$ by repeated ultrafiltration (Sartorius, $30 \mathrm{kD}$ ). Then, $100 \mu \mathrm{l}$ iodoacetamide (100 mM IAA in UA buffer) was added to block the reduced cysteine residues, and the samples were incubated for $30 \mathrm{~min}$ in darkness. The filters were washed with $100 \mu \mathrm{l}$ UA buffer three times and then $100 \mu \mathrm{l} 25 \mathrm{mM}$ NH4HCO3 buffer twice. Finally, the protein suspensions were digested with $4 \mu \mathrm{g}$ trypsin (Promega) in $40 \mu \mathrm{l} 25 \mathrm{mM}$ $\mathrm{NH} 4 \mathrm{HCO} 3$ buffer overnight at $37^{\circ} \mathrm{C}$. The resulting peptides were collected as a filtrate [24].

\section{Label-free LC-MS/MS}

Samples were analyzed on a nanoElute (Bruker, Bremen, Germany) coupled with a timsTOF Pro (Bruker, Bremen, Germany) and equipped with a CaptiveSpray source. Peptides were separated on a $25 \mathrm{~cm} \times 75 \mu \mathrm{m}$ analytical column and 1.6- $\mu \mathrm{m} \mathrm{C18}$ beads with a packed emitter tip (IonOpticks, Australia). The column temperature was maintained at $50^{\circ} \mathrm{C}$ using an integrated column oven (Sonation $\mathrm{GmbH}$, Germany). The column was equilibrated using four column volumes before loading the sample in $100 \%$ buffer A (99.9\% Milli-Q water, $0.1 \%$ FA); both steps were performed at 800 bar. Samples were separated at $300 \mathrm{~nL} / \mathrm{min}$ using a linear gradient from 2 to $25 \%$ buffer B (99.9\% ACN, 0.1\% FA) over 90 min before ramping to $37 \%$ buffer $\mathrm{B}(10 \mathrm{~min})$ and $80 \%$ buffer $\mathrm{B}$ $(10 \mathrm{~min})$ and was then sustained for $10 \mathrm{~min}$ (total separation method time: $120 \mathrm{~min}$ ) [25].

The timsTOF Pro (Bruker, Bremen, Germany) was operated in parallel accumulation-serial fragmentation (PASEF) mode. Mass range, $100-1700 \mathrm{~m} / \mathrm{z}$ (1/K0 Start $0.6 \mathrm{~V} \cdot \mathrm{s} / \mathrm{cm} 2$ and End $1.6 \mathrm{~V} \cdot \mathrm{s} / \mathrm{cm} 2)$; ramp time, $100 \mathrm{~ms}$; lock duty cycle, $100 \%$; capillary voltage, $1500 \mathrm{~V}$; dry gas, $3 \mathrm{~L} / \mathrm{min}$; dry temp, $180^{\circ} \mathrm{C}$; PASEF settings, $10 \mathrm{MS} /$ MS scans (total cycle time $=1.16 \mathrm{~s}$ ), charge range $=0-5$, active exclusion $=0.4 \mathrm{~min}$; scheduling target intensity, 20,000; intensity threshold, 2500; and CID collision energy, $42 \mathrm{eV}$. 


\section{Data analysis}

The MS data were analyzed using MaxQuant software version 1.6.14.0 [26]. An initial search was set at a precursor mass window of $6 \mathrm{ppm}$. MS data were searched against the Uniprot_MusMusculus_17027_20200226 database. The search followed an enzymatic cleavage rule of Trypsin/P and allowed a maximum of two missed cleavage sites and a mass tolerance of $20 \mathrm{ppm}$ for fragment ions. Carbamidomethylation of cysteines was defined as fixed modification, while protein $\mathrm{N}$-terminal acetylation and methionine oxidation were defined as variable modifications for database searching. The cut-off of global false discovery rate (FDR) for peptide and protein identification was set to 0.01 . Protein abundance was calculated on the basis of the normalized spectral protein intensity (LFQ intensity). Proteins with fold change $>2$ or $<0.5$ and $p$ value (Student's $t$-test) $<0.05$ were considered differentially expressed proteins [27-29].

\section{Western blotting}

Western blotting was performed as described previously [30]. For total protein extraction, mice livers were lysed by incubation with RIPA buffer containing protease inhibitor (Shenergy Biocolor Bioscience \&. Technology Company, Shanghai, China). Protein concentrations were determined using the BCA method. Equal amounts of protein were separated by $10 \%$ gel SDS-PAGE and electrophoretically transferred to a polyvinylidene difluoride membrane (Millipore, Darmstadt, Germany). The membranes were probed with primary antibodies overnight at $4{ }^{\circ} \mathrm{C}$, and incubated with the corresponding secondary antibodies. Immune complexes were measured through Enhanced Chemiluminescence Plus Detection System.

\section{Antibodies}

Anti-Bax antibody $((\mathrm{sc}-70,407)$ was purchased from Santa Cruz.

Biotechnology (Santa Cruz, CA). Anti-Deptor antibody (ab191841), anti-Stat1 antibody (ab140412), anti- $\beta$ actin antibody (ab6276), and GAPDH antibody were obtained from abcam (Cambridge, UK). $\beta$-tubulin antibody was from ABclonal (Wuhan, China). Anti-Rps6ka1 Polyclonal antibody was from Absin (Shanghai, China).

\section{Tunel assay}

Liver tissues from WT and LCN2-KO mice were formalin fixed and paraffin embedded. Apoptosis in paraffin-embedded sections was detected by terminal deoxynucleotidyl transferase (TdT)-mediated deoxyuridine triphosphate (dUTP) nick end labeling (Tunel) assay using a commercial kit (servicebio technology
CO.,LTD, Wuhan, China) according to the manufacturer's instructions. Briefly, the liver section was washed in 2 changes of xylene $15-20 \mathrm{~min}$ each, and followed by dehydrate in a gradient ethanol and distilled water. The section was incubated with proteinase $\mathrm{K}$ at $37^{\circ} \mathrm{C}$ for $25 \mathrm{~min}$ and permeabilized. After the slices are slightly dried, buffer is added to the tissues in the circle, and the buffer is incubated at room temperature for $10 \mathrm{~min}$. The tissue was incubated with appropriate amount of solution containing TdT, fluorescein (FITC)-labelled dUTP and buffer in a flat wet box for $120 \mathrm{~min}$ at $37^{\circ} \mathrm{C}$. The slides were washed with phosphate-buffered saline and incubated with diamidino-2-phenylindole (DAPI) solution at room temperature for $10 \mathrm{~min}$. Microscopic examination and collecting images was through fluorescence microscope. DAPI emits blue and FITC emits green, respectively.

\section{Gene Ontology (GO) analysis}

First, all protein sequences were aligned to the Homo sapiens database downloaded from NCBI (ncbiblast-2.2.28+-win32.exe), only the sequences in top 10 and E-value $<=1 \mathrm{e}-3$ were retained. Second, the GO term (database version: go_201504.obo) of the sequence was selected with top Bit-Score by Blast2GO [31]. Next, we completed the annotation from GO terms to proteins by Blast2GO Command Line. After the elementary annotation, InterProScan was used to search the EBI database by motifs and then, the functional information of motif to proteins was added to improve annotation. Then, further improvement of annotation and connection between GO terms was performed with ANNEX. Fisher's exact test was used to enrich the GO terms by comparing the number of differentially expressed proteins and total proteins correlated to GO terms [32]..

\section{Kyoto Encyclopedia of Genes and Genomes (KEGG) pathway annotation}

Pathway analysis was performed using the KEGG database. Fisher's exact test was used to identify the significantly enriched pathways by comparing the number of differentially expressed proteins and total proteins correlated to pathways.

\section{Results}

Differentially expressed apoptosis-associated proteins in the liver between LCN-KO mice and WT mice

To identify the proteins differentially expressed in the liver of LCN-KO mice, liver extracts from LCN2-KO and WT mice were comparatively analyzed by labelfree quantitative proteomics. The proteomics analysis 


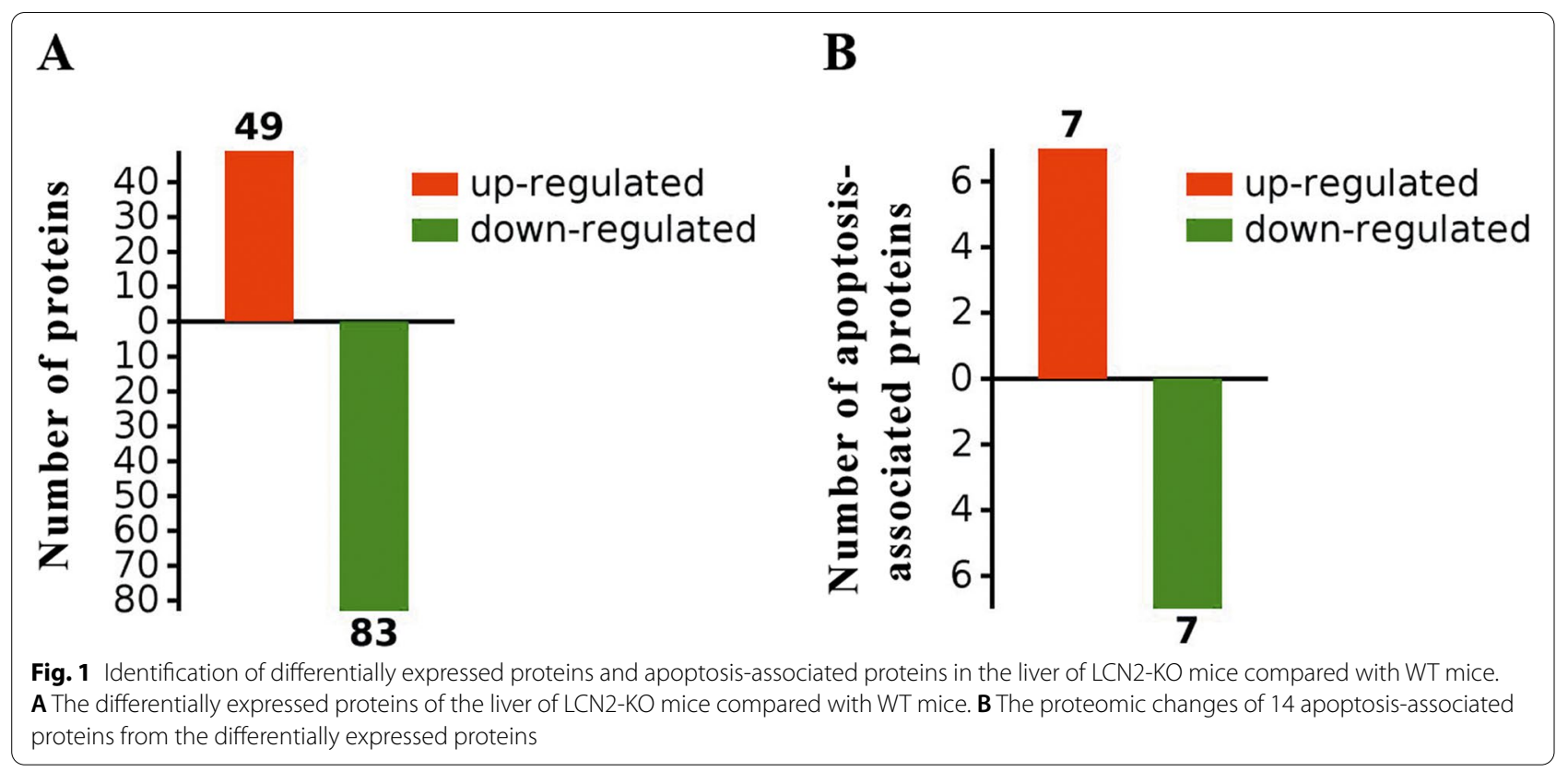

revealed 132 significantly differentially expressed proteins (49 upregulated and 83 downregulated [fold change $\geq 2, P<0.05])$ among the 2140 proteins that had quantification information (data not shown) in the liver of LCN2-KO mice compared with WT mice (Fig. 1A). Of these, seven apoptosis-associated proteins were significantly upregulated and seven apoptosis-associated proteins downregulated (Fig. 1B). A list of 259 apoptosis-associated proteins with their fold changes and 14 differentially expressed proteins are shown in Table 1 and supplementary file.

\section{Bioinformatics analysis of differentially expressed apoptosis-associated proteins}

To further investigate the effect of LCN2 on the apoptotic process, we analyzed the proteomic data using GO analysis and KEGG pathway enrichment analysis. As shown in Fig. 2, 17\% of all identified differentially expressed proteins were implicated in peptide transport process, and $13 \%$ of proteins were involved in the monocarboxylic acid metabolic process. Fourteen differentially expressed proteins in the liver of LCN2-KO mice were likely associated with the regulation of the neuronal apoptotic processes.

Table 1 Identification of differentially expressed apoptosis-associated proteins in the liver of LCN2-KO mice compared with WT mice

\begin{tabular}{lllll}
\hline Change & Protein IDs & Protein Name & Gene Name & Fold Change \\
\hline up & Q07813 & Apoptosis regulator BAX & Bax & Hcls1 \\
up & P49710 & Hematopoietic lineage cell-specific protein & Deptor & 3.37 \\
up & Q570Y9 & DEP domain-containing mTOR-interacting protein & Ggct & 3.14 \\
up & Q9D7X8 & Gamma-glutamyl cyclotransferase & Anxa1 & 2.93 \\
up & P10107 & Annexin A1 & Dnaja3 & 2.56 \\
up & Q99M87 & DnaJ homolog subfamily A member 3 & Cul3 & 2.39 \\
up & Q9JLV5 & Cullin-3 & Stat1 & 2.25 \\
down & P42225 & Signal transducer and activator of transcription 1 & Rps6ka1 & 0.49 \\
down & P18653 & Ribosomal protein S6 kinase alpha-1 & Sirt2 & 0.47 \\
down & Q8VDQ8 & NAD-dependent protein deacetylase sirtuin-2 & Ube2m & 0.36 \\
down & P61082 & NEDD8-conjugating enzyme Ubc12 & Anp32b \\
down & Q9EST5 & Acidic leucine-rich nuclear phosphoprotein 32 family mem- & & 0.24 \\
down & O88738 & ber B & Birc6 & 0.22 \\
down & Q9JIZ0 & Baculoviral IAP repeat-containing protein 6 & Cml1 & 0.16
\end{tabular}




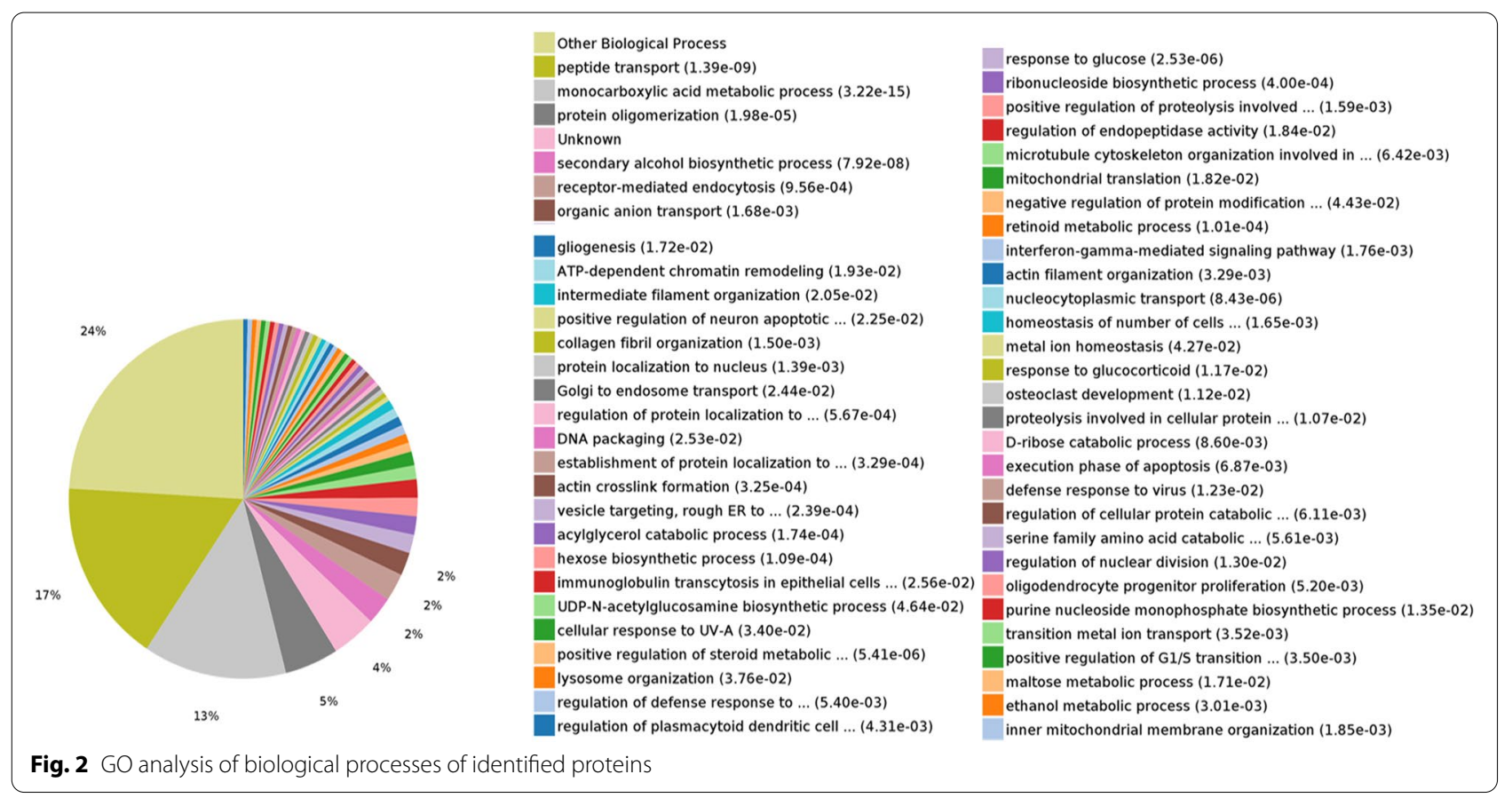

KEGG analysis revealed that $8 \%$ differentially expressed proteins were associated with metabolic pathways; these indicated that LCN2 played an important role in metabolism. According to the above-mentioned results, several differentially expressed proteins were linked to regulating apoptotic process (Fig. 3).

To confirm the effect of differentially expressed proteins on apoptosis in LCN2-KO mice, western blotting was used to detect the 4 significantly changed proteins, and Tunel assay was performed to evaluate the apoptotic process in the liver of LCN2-KO mice. In consistent with proteomics result (Table 1), we also observed an obvious increase of apoptosis regulator BAX (Bax) and DEP domain-containing mTOR-interacting protein (Deptor) protein level, and an obvious decrease of signal transducer and activator of transcription 1 (Stat1) and Ribosomal protein S6 kinase alpha-1 (Rps6ka1) protein level. Tunel assay suggested evidence of increased apoptosis in the liver of LCN2-KO mice (Fig. 4). We visualized 14 differentially expressed apoptosis-associated proteins using a heatmap (Fig. 5). To better understand the regulatory role of 14 differentially expressed apoptotic proteins, we further established a network of protein-protein interactions (PPIs) between the identified differentially apoptotic proteins and other possible factors (Fig. 6). Various apoptosis-associated proteins interacted with each other and formed a complicated network suggesting a complex regulatory mechanism.

\section{Discussion}

LCN2 is primarily an iron-trafficking protein involved in the regulation of cellular iron ion homeostasis, and recent studies have shown that LCN2 had multiple functions involved in various biological and pathological processes including energy homeostasis, cancer, inflammation, and apoptosis [3, 8, 12, 19, 35].

Several studies have reported that LCN2 protein expression is upregulated in human diseases such as obesity, type 2 diabetes, and a wide range of cancers $[8,12,15,36]$. Many studies have investigated and revealed the underlying mechanisms implicated in multiple pathways [12, 37]. Downregulation of LCN2 has been reported in oral squamous cell carcinoma [38]. Law et al. demonstrated that insulin resistance and obesity could be improved in mice with $L C N 2$ gene $\mathrm{KO}$, suggesting LCN2 to be a risk factor, wherein the study by Guo et al. showed that insulin resistance and obesity could be potentiated in mice with $L C N 2$ gene $K O$, suggesting $L C N 2$ to be a protective factor $[16$, 39]. These suggest that some contradictory findings need to be further investigated and addressed.

Apoptosis played an important role in human disease processes such as cancer, autoimmune disease, metabolic disease, and ageing $[8,40,41]$. In our study, we showed that 14 differentially expressed proteins were related with apoptotic processes in the liver of LCN2-KO mice suggesting that LCN2-induced apoptosis was critical for regulation of cellular biological processes.

Proteomics demonstrated that there were seven upregulated apoptosis-associated proteins mediated by $L C N 2$ 


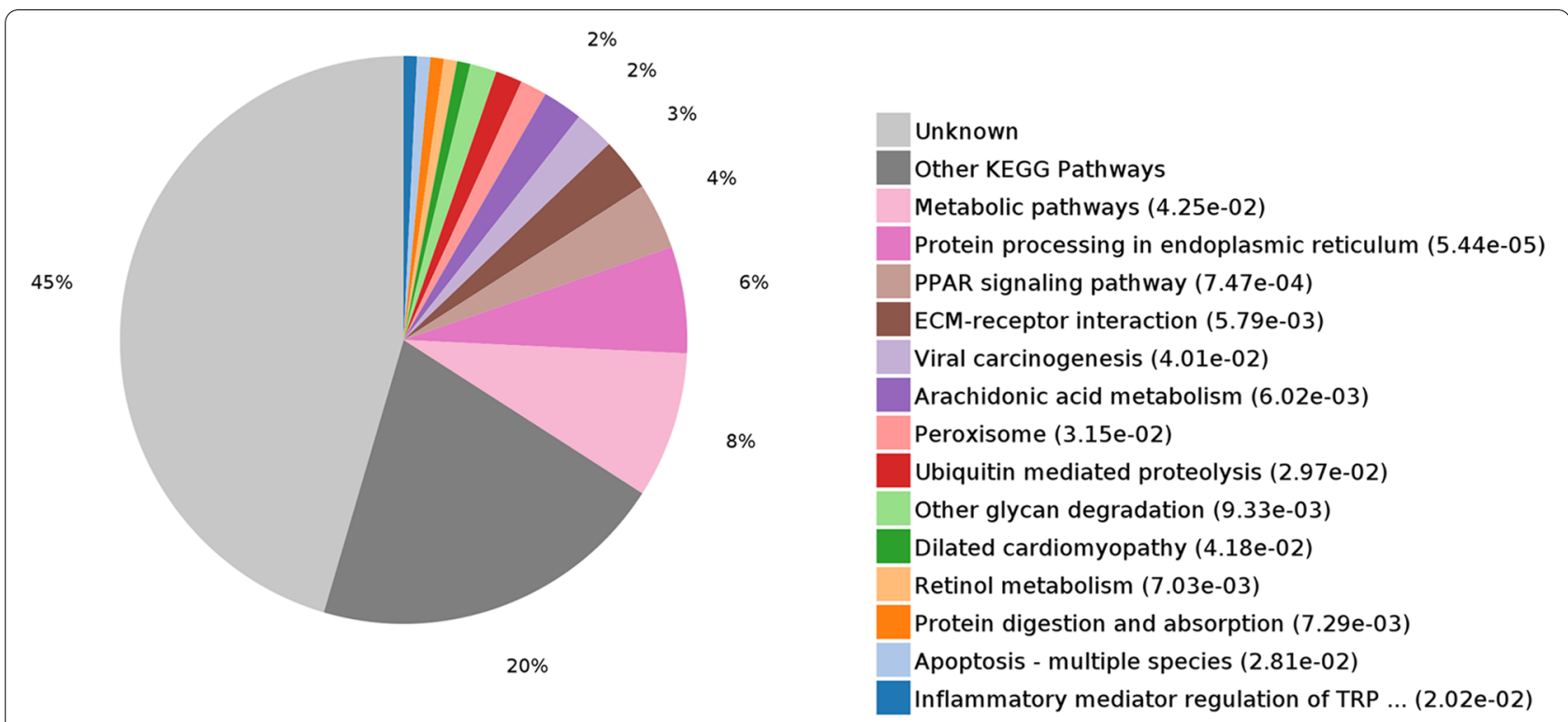

Fig. 3 KEGG pathway analysis of differentially expressed proteins

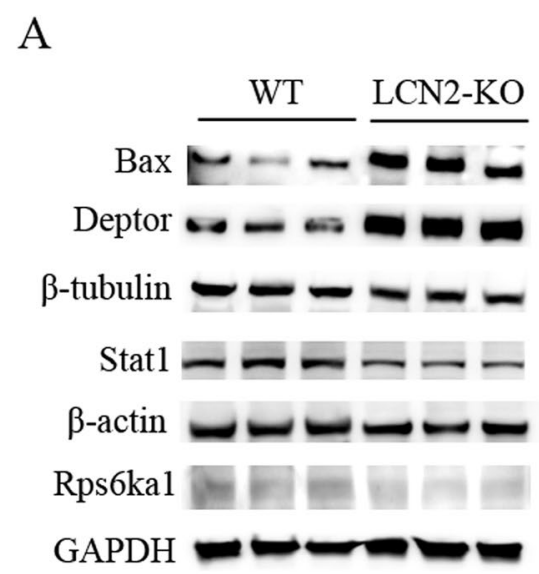

B

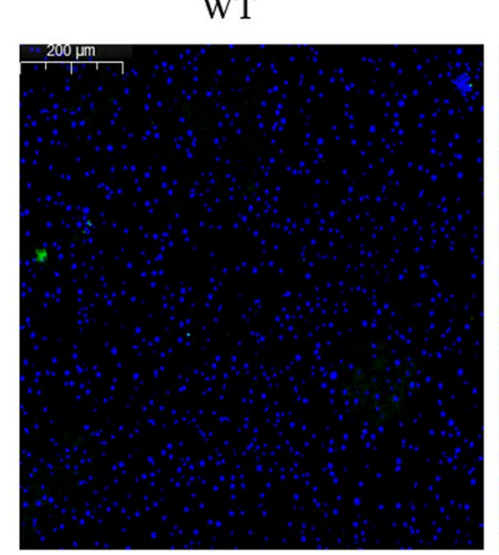

LCN2-KO

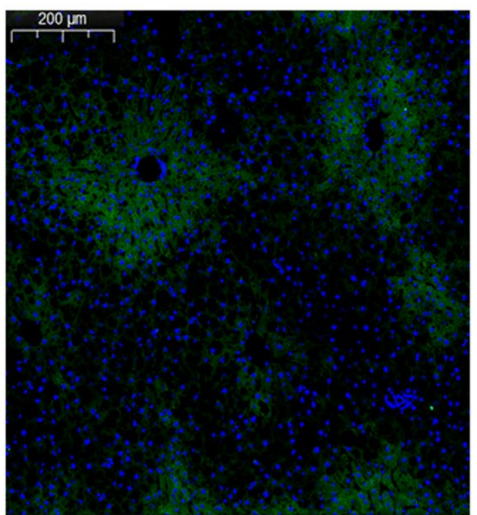

Fig. 4 Apoptosis was evaluated using the Tunel assay, DAPI nuclear staining, and Western blotting analysis. A. Upregulation of Bax and Deptor protein, and downregulation of Stat1 and Rps6ka1 protein in the livers of the LCN2-KO mice were determined by western blotting. Experiments were conducted in triplicates. Full-length blots/gels were presented in Supplementary file Fig. S1, S2, S3, S4, S5, S6 and S7. B. Representative images of the Tunel assay in the livers of the WT and LCN2-KO mice. Nucleus is blue by labeling with DAPI. Positive apoptosis cells are green. Scale bar, $200 \mu \mathrm{m}$. Abbreviations: Bax: apoptosis regulator BAX. Deptor: DEP domain-containing mTOR-interacting protein. Stat1: signal transducer and activator of transcription 1. Rps6ka1: Ribosomal protein 56 kinase alpha-1

in the mouse liver. A previous study has revealed that LCN2 induced apoptosis by inhibiting B-cell lymphoma 2 (Bcl-2)-associated agonist of cell death (Bad) and another $\mathrm{Bcl}-2$ family member, $\left(\mathrm{Bcl}-\mathrm{X}_{\mathrm{L}}\right)$, under the condition of interleukin-3 (IL-3) deprivation in FL5.12 lymphocytic cells [4]. In our study, the levels of Bad and Bcl-X $\mathrm{L}_{\mathrm{L}}$ proteins and that of $\mathrm{Bcl}-2$-associated transcription factor 1 (Btf) and Bcl-2-like protein 13 (Bcl2-L-13) did not significantly change (supplementary file). Proteomics analysis showed that seven differentially expressed apoptosis-associated proteins were upregulated in the liver of LCN2-KO mice. Apoptosis regulator BAX (Bax) could activate caspase- 3 and the apoptotic process under stress conditions involved in the regulation of apoptotic signaling pathway interaction with Bcl-2 or Bcl- $\mathrm{X}_{\mathrm{L}}$ [42]. Our study showed that Bax was a pro-apoptotic protein and was significantly upregulated by 5.4 -fold suggesting that LCN2 was an important regulatory molecule of apoptotic signaling pathway in the liver. 


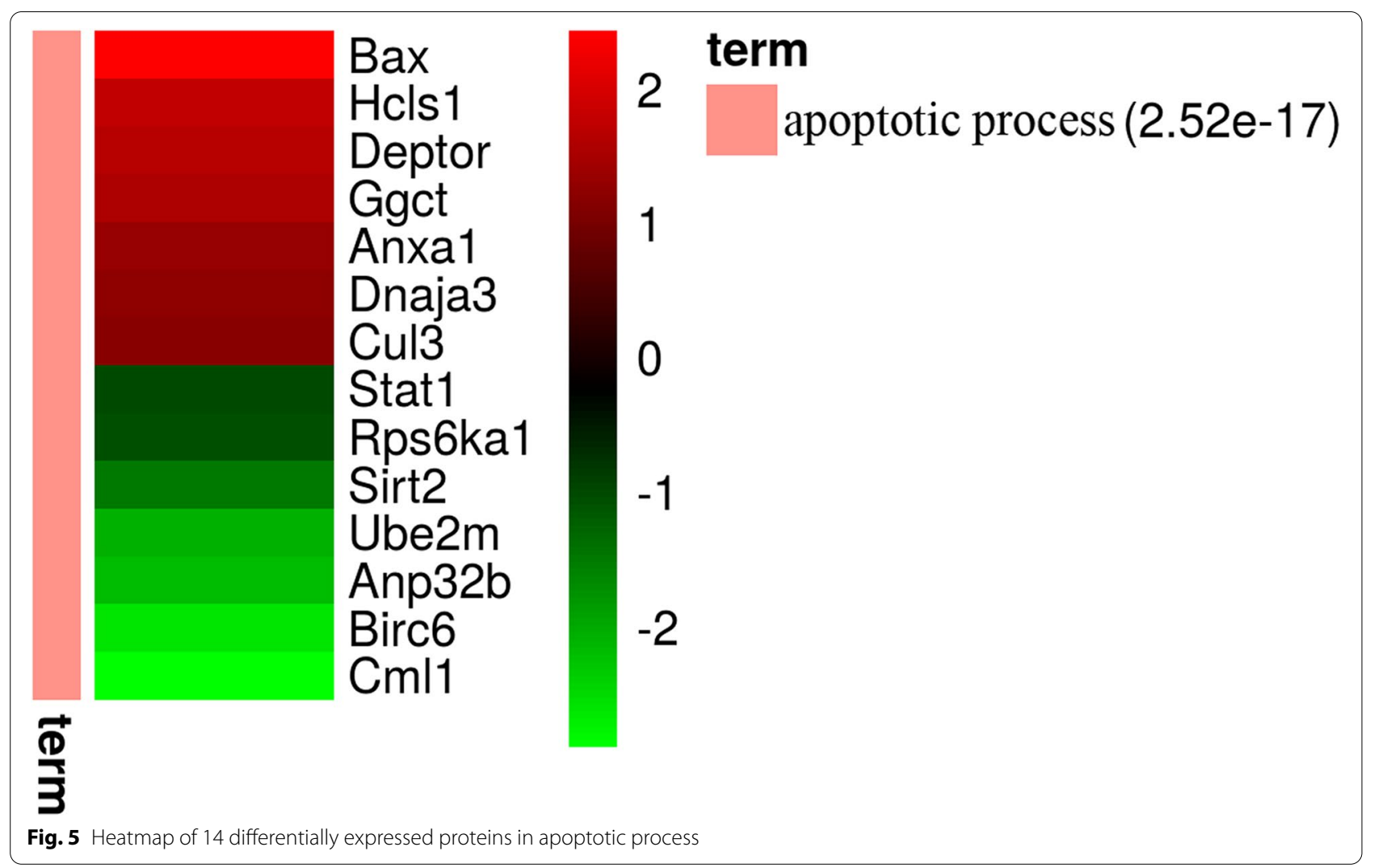

The hematopoietic lineage cell-specific protein (Hlcs1) is a substrate of the antigen receptor-coupled tyrosine kinase and plays a role in B lymphocyte cell antigen receptor (BCR)-mediated apoptosis and the regulation of tyrosine phosphorylation of signal transducer and activator of transcription (Stat) protein [43, 44]. DEP domain-containing mTOR-interacting protein (Deptor) was physiologically involved in the inhibition of mammalian target of rapamycin (mTOR) signaling pathway and induced apoptosis [45]. Gamma-glutamyl cyclotransferase (Ggct) induced apoptosis by promoting the release of cytochrome $\mathrm{C}$ from mitochondria [46]. Future studies should investigate the physiological significance of these three upregulated proteins in LCN2-KO mice.

The DnaJ homolog subfamily A member 3 (Dnaja3) regulates apoptotic signal transduction by influencing cytochrome $\mathrm{C}$ release from the mitochondria and activating caspase 3 [47]. Dnaja3 protein was upregulated in the hippocampi of Alzheimer's disease (AD) patients and the AD mouse model, and Dnaja3 induced amyloid $\beta 42$ (A $\beta 42)$ production and neuronal apoptosis, which played a crucial role in the pathogenesis of $\operatorname{AD}[48,49]$. Recently, a growing body of evidence has indicated that the LCN2 was associated with AD pathogenesis, vascular dementia, and other neurodegenerative dementias $[50,51]$. There was a significant increase of Dnaja3 in the liver of LCN2-KO mice indicating a close association between LCN2 and Dnaja3. The GO analysis revealed that LCN2 was involved in positive regulation of neuronal apoptosis. However, despite no detection of LCN2 in the neurons of LCN2-KO mice, we speculate that Dnaja3 is likely an important mediator of LCN2-induced apoptosis in nervous system diseases.

Our data revealed that seven apoptosis-associated proteins were obviously decreased in the liver of LCN2-KO mice, in which Stat1 was the most drastically decreased. Stat 1 mediates multiple biological processes including cell development, angiogenesis and insulin signal pathway, and stimulates caspase activity and IFN-induced apoptosis [52, 53]. Early studies have demonstrated that IFN $\gamma$ can activate Stat1 and Stat 1 can directly bind to the promoter of $L C N 2$ gene and upregulate its expression in adipocytes, which suggested that Stat1 likely played a critical role in LCN2's apoptotic function $[54,55]$. Stat 1 also interacted with Dnaja3 to influence IFNY-indued apoptosis (Fig. 6) [56]. Therefore, numerous proteins interacted with other regulators to represent different effects on biological functions such as inhibition or activation.

Ribosomal protein S6 kinase alpha-1 (Rps6ka1) is a serine/threonine-protein kinase that regulates apoptosis by phosphorylating $\mathrm{Bad}$ and repressing $\mathrm{Bad}$ 


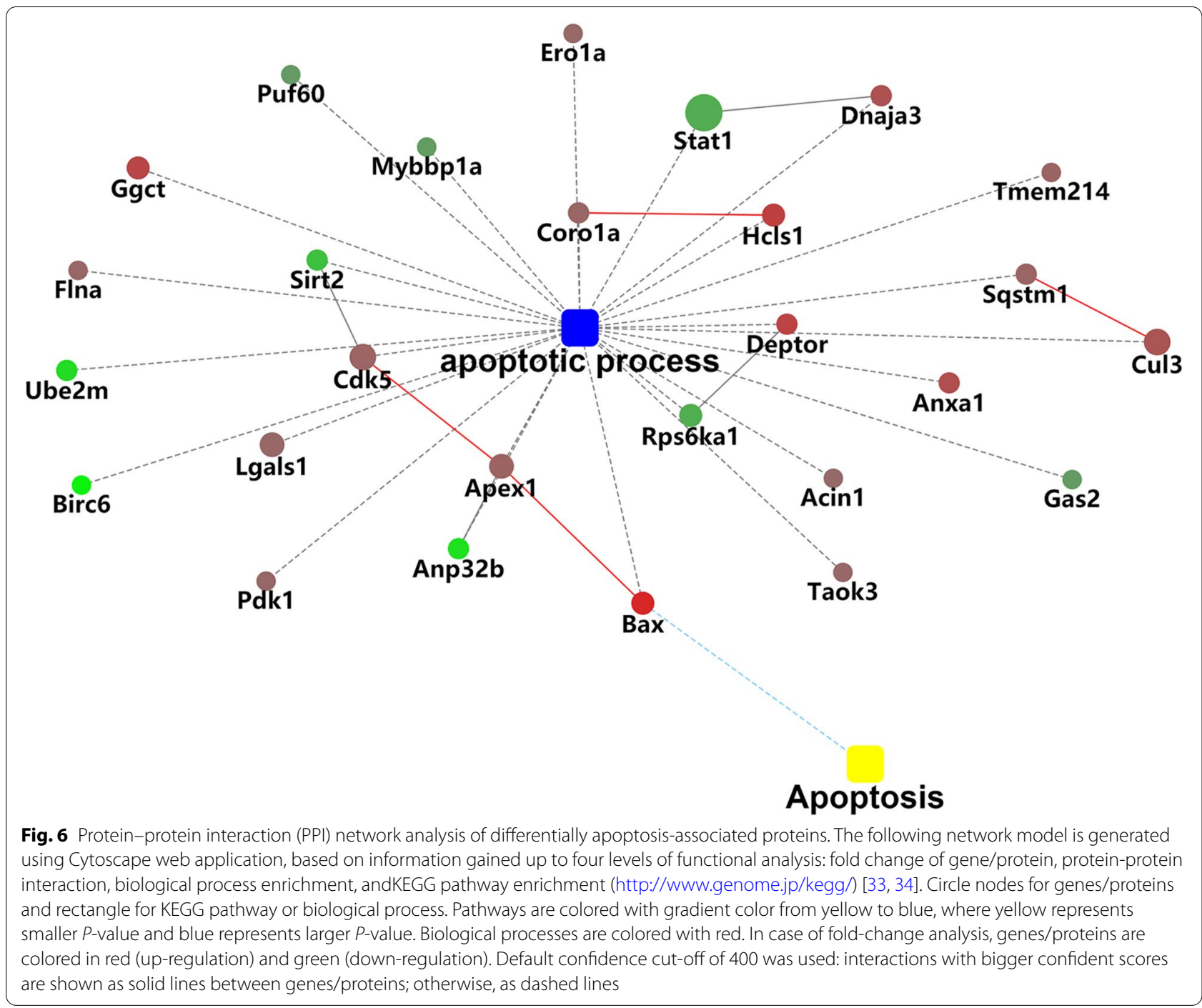

function [57, 58]. Consistent with previous findings by Devireddy et al., LCN2 possibly induced apoptosis through Rps6ka1-mediated phosphorylation of Bad [4].

NAD-dependent protein deacetylase sirtuin-2 (Sirt2) deacetylates downstream target proteins to modulate diverse cellular processes such as cell cycle, apoptosis, differentiation, metabolism, and autophagy [59]. Sirt2 reduces oxidative stress-induced apoptosis by influencing the cMYC (a transcription factor) pathway in cholangiocarcinoma [60]. However, according to present literature, it has not been reported in the direct interaction of LCN2 and Sirt2.

NEDD8-conjugating enzyme Ubc12 (Ube2m) interacts with E3 ubiquitin ligase RBX1 to play a role in the positive regulation of neuron apoptotic processes and cell proliferation through neddylation modification of Cullin3 (Cul3) [61, 62]. The interaction of Ube2m and Cul3 might be associated with the LCN2-mediated regulation of neuronal apoptosis (Fig. 2). Nonetheless, the exact mechanism of LCN2-mediated apoptosis through these apoptosis proteins or interaction is still not well studied and needs further investigation.

Our study focused on the effect of LCN2 on apoptosis which is involved in the pathogenesis of insulin resistance, cancer, and nervous system diseases. Our results might provide new insights in the development of novel therapeutic targets for metabolic diseases and/or cancer. A limitation of the study was the lack of validation of the identified upregulated and downregulated apoptosis-associated proteins. Further studies are required to investigate the physiological significance of these LCN2-mediated apoptotic proteins in the liver and other tissues. 


\section{Conclusion}

Our proteomics analysis has identified seven upregulated and seven downregulated apoptosis-associated proteins in the liver of $\mathrm{LCN} 2-\mathrm{KO}$ mice. It is vital to clarify the effect of LCN2 protein on apoptosis as the latter likely contributes to the pathogenesis of insulin resistance, cancer, and various nervous system diseases.

\section{Supplementary Information}

The online version contains supplementary material available at https://doi. org/10.1186/s12864-021-08211-y.

Additional file 1: Figure S1. Full-length blot of Bax protein expression level. Figure. S2. Full-length blot of Deptor protein expression level. Figure. S3. Full length blot of $\beta$-tubulin protein expression level. Figure S4. Full-length blot of Stat1 protein expression level. Figure S5. Fulllength blot $\beta$-actin protein expression level. Figure S6. Full-length blot of Rps6ka1 protein expression level. Figure S7. Full length blot GAPDH protein expression level.

Additional file 2.

\section{Acknowledgements}

We thank the animal technicians of scientific centre of Qingdao Hospital of Traditional Chinese Medicine (Qingdao, China) for their skillful assistance during this study.

\begin{abstract}
Authors' contributions
DMW was responsible for the data analysis and writing the paper. XPW and YH carried out the animal experiments and proteomics analysis. YYW was responsible for the overall planning, design of the study, interpretation of data, and revision of the manuscript. All authors read and approved the final manuscript.
\end{abstract}

\section{Funding}

No.

\section{Availability of data and materials}

All data generated and analyzed during our study are included in the published article and supplied in one supplementary file.

\section{Declarations}

\section{Ethics approval and consent to participate}

This study was approved by the Ethics Committee of Qingdao Hospital of Traditional Chinese Medicine (No.2020HC12LQ096). All methods were carried out in accordance with relevant guidelines and regulations. This study was carried out in compliance with the ARRIVE guidelines.

\section{Consent for publication}

Not applicable.

\section{Competing interests}

The authors report no conflicts of interest in this work.

Received: 25 May 2021 Accepted: 23 November 2021

Published online: 13 December 2021

\section{References}

1. Cowland JB, Borregaard N. Molecular characterization and pattern of tissue expression of the gene for neutrophil Gelatinase-associated Lipocalin from humans. Genomics. 1997;45(1):17-23. https://doi.org/10.1006/geno. 1997.4896.
2. Shields-Cutler RR, Crowley JR, Miller CD, Stapleton AE, Cui W, Henderson JP. Human Metabolome-derived cofactors are required for the antibacterial activity of Siderocalin in urine. J Biol Chem. 2016;291(50):25901-10. https://doi.org/10.1074/jbc.M116.759183.

3. Yang J, Goetz D, Li J-Y, Wang W, Mori K, Setlik D, et al. An Iron delivery pathway mediated by a Lipocalin. Mol Cell. 2002;10(5):1045-56. https:// doi.org/10.1016/s1097-2765(02)00710-4.

4. Devireddy LR. Induction of apoptosis by a secreted Lipocalin that is transcriptionally regulated by IL-3 deprivation. Science. 2001;293(5531):82934. https://doi.org/10.1126/science.1061075.

5. Holmes MA, Paulsene W, Jide X, Ratledge C, Strong RK. Siderocalin (Lcn 2) also binds carboxymycobactins, potentially defending against mycobacterial infections through iron sequestration. Structure. 2005;13(1):29-41. https://doi.org/10.1016/j.str.2004.10.009.

6. Fagerberg L, Hallstrom BM, Oksvold P, Kampf C, Djureinovic D, Odeberg J, et al. Analysis of the human tissue-specific expression by genome-wide integration of Transcriptomics and antibody-based proteomics. Mol Cell Proteomics. 2014;13(2):397-406. https://doi.org/10.1074/mcp.M113.035600.

7. Xu M-J, Feng D, Wu H, Wang H, Chan Y, Kolls J, et al. Liver is the major source of elevated serum lipocalin-2 levels after bacterial infection or partial hepatectomy: a critical role for IL-6/STAT3. Hepatology. 2015;61(2):692-702. https://doi.org/10.1002/hep.27447.

8. Wen X, Su B, Gao M, Chen J, Zhou D, You H, et al. Obesity-associated up-regulation of lipocalin 2 protects gastric mucosa cells from apoptotic cell death by reducing endoplasmic reticulum stress. Cell Death Dis. 2021;12(2). https://doi.org/10.1038/s41419-021-03512-2.

9. Ota T, Suzuki Y, Nishikawa T, Otsuki T, Sugiyama T, Irie R, et al. Complete sequencing and characterization of 21,243 full-length human cDNAs. Nat Genet. 2003;36(1):40-5. https://doi.org/10.1038/ng1285.

10. Gumper K, Dangel AW, Pita-Grisanti V, Krishna SG, Lara LF, Mace T, et al. Lipocalin-2 expression and function in pancreatic diseases. Pancreatology. 2020;20(3):419-24. https://doi.org/10.1016/j.pan.2020.01.002.

11. Xu Y, Zhu Y, Jadhav K, Li Y, Sun H, Yin L, et al. Lipocalin-2 protects against diet-induced nonalcoholic fatty liver disease by targeting hepatocytes. Hepatol Commun. 2019;3(6):763-75. https://doi.org/10.1002/hep4.1341.

12. Rahimi S, Roushandeh AM, Ahmadzadeh E, Jahanian-Najafabadi A, Roudkenar MH. Implication and role of neutrophil gelatinase-associated lipocalin in cancer: lipocalin-2 as a potential novel emerging comprehensive therapeutic target for a variety of cancer types. Mol Biol Rep. 2020;47(3):2327-46. https://doi.org/10.1007/s11033-020-05261-5.

13. Huang Y, Yang Z, Ye Z, Li Q, Wen J, Tao X, et al. Lipocalin-2, glucose metabolism and chronic low-grade systemic inflammation in Chinese people. Cardiovasc Diabetol. 2012;11(1):11. https://doi.org/10.1186/ 1475-2840-11-11.

14. Ishii A, Katsuura G, Imamaki H, Kimura H, Mori KP, Kuwabara T, et al. Obesity-promoting and anti-thermogenic effects of neutrophil gelatinaseassociated lipocalin in mice. Sci Rep. 2017;7(1). https://doi.org/10.1038/ s41598-017-15825-4.

15. Rashad NM, El-Shal AS, Etewa RL, Wadea FM. Lipocalin-2 expression and serum levels as early predictors of type 2 diabetes mellitus in obese women. IUBMB Life. 2017;69(2):88-97. https://doi.org/10.1002/iub.1594

16. Law IKM, Xu A, Lam KSL, Berger T, MakTW, Vanhoutte PM, et al. Lipocalin-2 deficiency attenuates insulin resistance associated with aging and obesity. Diabetes. 2010;59(4):872-82. https://doi.org/10.2337/db09-1541.

17. Chung $\mathrm{IH}$, Chen $\mathrm{C}-\mathrm{Y}$, Lin $\mathrm{Y}-\mathrm{H}$, Chi H-C, Huang Y-H, Tai P-J, et al. Thyroid hormone-mediated regulation of lipocalin 2 through the met/FAK pathway in liver cancer. Oncotarget. 2015;6(17):15050-64. https://doi.org/10. 18632/oncotarget.3670.

18. Zhang Y, Fan Y, Mei Z. NGAL and NGALR overexpression in human hepatocellular carcinoma toward a molecular prognostic classification. Cancer Epidemiol. 2012;36(5):e294-9. https://doi.org/10.1016/j.canep. 2012.05.012.

19. Lin H-H, Liao C Jr, Lee Y-C, Hu K-H, Meng H-W, Chu S-T. Lipocalin2-induced cytokine production enhances endometrial carcinoma cell survival and migration. Int J Biol Sci. 2011;7(1):74-86. https://doi.org/10. 7150/ijbs.7.74.

20. Rahimi S, Roushandeh AM, Ebrahimi A, Samadani AA, Kuwahara Y, Roudkenar MH. CRISPR/Cas9-mediated knockout of Len2 effectively enhanced CDDP-induced apoptosis and reduced cell migration capacity of PC3 cells. Life Sci. 2019;231:116586. https://doi.org/10.1016/j.lfs.2019. 116586. 
21. Berger T, Togawa A, Duncan GS, Elia AJ, You-Ten A, Wakeham A, et al. Lipocalin 2-deficient mice exhibit increased sensitivity to Escherichia coli infection but not to ischemia-reperfusion injury. Proc Natl Acad Sci. 2006;103(6):1834-9. https://doi.org/10.1073/pnas.0510847103.

22. Zhu Y, Xu H, Chen H, Xie J, Shi M, Shen B, et al. Proteomic analysis of solid Pseudopapillary tumor of the pancreas reveals dysfunction of the endoplasmic reticulum protein processing pathway. Mol Cell Proteomics. 2014;13(10):2593-603. https://doi.org/10.1074/mcp.M114.038786.

23. Rashid ST, Humphries JD, Byron A, Dhar A, Askari JA, Selley JN, et al. Proteomic analysis of extracellular matrix from the hepatic stellate cell line LX-2 identifies CYR61 and Wnt-5a as novel constituents of fibrotic liver. Proteome Res. 2012;11(8):4052-64. https://doi.org/10.1021/pr3000927.

24. Wiśniewski JR, Zougman A, Nagaraj N, Mann M. Universal sample preparation method for proteome analysis. Nat Methods. 2009;6(5):359-62. https://doi.org/10.1038/nmeth.1322.

25. Levin Y, Schwarz E, Wang L, Leweke FM, Bahn S. Label-free LC-MS/MS quantitative proteomics for large-scale biomarker discovery in complex samples. J Sep Sci. 2007;30(14):2198-203. https://doi.org/10.1002/jssc. 200700189.

26. Cox J, Mann M. MaxQuant enables high peptide identification rates, individualized p.p.b.-range mass accuracies and proteome-wide protein quantification. Nat Biotechnol. 2008;26(12):1367-72. https://doi.org/10. 1038/nbt.1511.

27. Zybailov B, Coleman MK, Florens L, Washburn MP. Correlation of relative abundance ratios derived from peptide ion chromatograms and Spectrum counting for quantitative proteomic analysis using stable isotope labeling. Anal Chem. 2005;77(19):6218-24. https://doi.org/10.1021/ac050 $846 r$.

28. Pinto SM, Manda SS, Kim M-S, Taylor K, Selvan LDN, Balakrishnan L, et al. Functional annotation of proteome encoded by human chromosome 22. J Proteome Res. 2014;13(6):2749-60. https://doi.org/10.1021/pr401169d.

29. Toraskar J, Magnussen SN, Hagen L, Sharma A, Hoang L, Bjørkøy G, et al. A novel truncated form of Nephronectin is present in small extracellular vesicles isolated from 66cl4 cells. J Proteome Res. 2019;18(3):1237-47. https://doi.org/10.1021/acs.jproteome.8b00859.

30. Kim S-L, Min IS, Park YR, Lee ST, Kim S-W. Lipocalin 2 inversely regulates TRAIL sensitivity through p38 MAPK-mediated DR5 regulation in colorectal cancer. Int J Oncol. 2018. https://doi.org/10.3892/ijo.2018.4562.

31. Gotz S, Garcia-Gomez JM, Terol J, Williams TD, Nagaraj SH, Nueda MJ, et al. High-throughput functional annotation and data mining with the Blast2GO suite. Nucleic Acids Res. 2008;36(10):3420-35. https://doi.org/ 10.1093/nar/gkn176.

32. Wang $X$, Shi Y, He R, Li B, Huang A. Label-free quantitative proteomic analysis of the biological functions of Moringa oleifera seed proteins provides insights regarding the milk-clotting proteases. Int J Biol Macromol. 2020;144:325-33. https://doi.org/10.1016/j.ijbiomac.2019.12.070.

33. Kanehisa M. KEGG: Kyoto encyclopedia of genes and genomes. Nucleic Acids Res. 2000;28(1):27-30. https://doi.org/10.1093/nar/28.1.27.

34. Kanehisa M. Toward understanding the origin and evolution of cellular organisms. Protein Sci. 2019;28(11):1947-51. https://doi.org/10.1002/pro. 3715.

35. Asimakopoulou A, Weiskirchen S, Weiskirchen R. Lipocalin 2 (LCN2) expression in hepatic malfunction and therapy. Front Physiol. 2016;7. https://doi.org/10.3389/fphys.2016.00430.

36. Rahman MM, Hasan MM, MasudRana HI, Rahman MS, Asaduzzaman M, Munira S, et al. Elevated serum lipocalin-2 is a risk factor of insulin resistance and for the progression of impaired glucose to type 2 diabetes in Bangladeshi population. Int J Pharm Technol. 2015;7(3):9785-94.

37. Moschen AR, Adolph TE, Gerner RR, Wieser V, Tilg H. Lipocalin-2: a master mediator of intestinal and metabolic inflammation. Trends Endocrinol Metab. 2017;28(5):388-97. https://doi.org/10.1016/j.tem.2017.01.003.

38. Monisha J, Roy N, Padmavathi G, Banik K, Bordoloi D, Khwairakpam A, et al. NGAL is Downregulated in Oral squamous cell carcinoma and leads to increased survival, proliferation, Migration and Chemoresistance. Cancers. 2018;10(7):228. https://doi.org/10.3390/cancers10070228.

39. Guo H, Jin D, Zhang Y, Wright W, Bazuine M, Brockman DA, et al. Lipocalin-2 deficiency impairs thermogenesis and potentiates diet-induced insulin resistance in mice. Diabetes. 2010;59(6):1376-85. https://doi.org/ 10.2337/db09-1735

40. Wong RSY. Apoptosis in cancer: from pathogenesis to treatment. J Exp Clin Cancer Res. 2011;30(1). https://doi.org/10.1186/1756-9966-30-87.
41. Argüelles S, Guerrero-Castilla A, Cano M, Muñoz MF, Ayala A. Advantages and disadvantages of apoptosis in the aging process. Ann N Y Acad Sci. 2019;1443(1):20-33. https://doi.org/10.1111/nyas.14020.

42. Edlich F, Banerjee S, Suzuki M, Cleland Megan M, Arnoult D, Wang C, et al. $\mathrm{BCl}-\mathrm{xL}$ Retrotranslocates Bax from the mitochondria into the cytosol. Cell. 2011;145(1):104-16. https://doi.org/10.1016/j.cell.2011.02.034.

43. Yamanashi Y, Fukuda T, Nishizumi H, Inazu T, Higashi K-i, Kitamura D, et al. Role of tyrosine phosphorylation of HS1 in B cell antigen receptor-mediated apoptosis. J Exp Med. 1997;185(7):1387-92. https://doi.org/10.1084/ jem.185.7.1387.

44. Lu X, Chen J, Sasmono RT, Hsi ED, Sarosiek KA, Tiganis T, et al. T-cell protein tyrosine phosphatase, distinctively expressed in activated-B-cell-like diffuse large B-cell lymphomas, is the nuclear phosphatase of STAT6. Mol Cell Biol. 2007;27(6):2166-79. https://doi.org/10.1128/MCB.01234-06.

45. Peterson TR, Laplante M, Thoreen CC, Sancak Y, Kang SA, KuehI WM, et al. DEPTOR is an mTOR inhibitor frequently overexpressed in multiple myeloma cells and required for their survival. Cell. 2009;137(5):873-86. https://doi.org/10.1016/j.cell.2009.03.046.

46. Masuda Y, Maeda S, Watanabe A, Sano Y, Aiuchi T, Nakajo S, et al. A novel 21-kDa cytochrome c-releasing factor is generated upon treatment of human leukemia U937 cells with geranylgeraniol. Biochem Biophys Res Commun. 2006;346(2):454-60. https://doi.org/10.1016/j.bbrc.2006.05.161.

47. Syken J, De-Medina T, Munger K. TID1, a human homolog of the Drosophila tumor suppressor I(2)TID, encodes two mitochondrial modulators of apoptosis with opposing functions. Proc Natl Acad Sci U S A. 1999;96(15):8499-504. https://doi.org/10.1073/pnas.96.15.8499.

48. Zhou C, Taslima F, Abdelhamid M, Kim S-W, Akatsu H, Michikawa M, et al. Beta-amyloid increases the expression levels of Tid1 responsible for neuronal cell death and amyloid Beta production. Mol Neurobiol. 2019;57(2):1099-114. https://doi.org/10.1007/s12035-019-01807-2.

49. Karran E, Mercken M, Strooper BD. The amyloid cascade hypothesis for Alzheimer's disease: an appraisal for the development of therapeutics. Nat Rev Drug Discov. 2011;10(9):698-712. https://doi.org/10.1038/nrd35 05

50. Song J, Kim OY. Perspectives in Lipocalin-2: emerging biomarker for medical diagnosis and prognosis for Alzheimer's disease. Clin Nutr Res. 2018;7(1):1. https://doi.org/10.7762/cnr.2018.7.1.1.

51. Llorens F, Hermann P, Villar-Piqué A, Diaz-Lucena D, Nägga K, Hansson O, et al. Cerebrospinal fluid lipocalin 2 as a novel biomarker for the differential diagnosis of vascular dementia. Nature. Communications. 2020;11(1). https://doi.org/10.1038/s41467-020-14373-2.

52. Huang Y-Q, Li J-J, Karpatkin S. Thrombin inhibits tumor cell growth in association with up-regulation of p21 and Caspases via a p53-independent, STAT-1-dependent pathway. J Biol Chem. 2000;275(9):6462-8. https://doi.org/10.1074/jbc.275.9.6462.

53. Meng C, Guo L-b, Liu X, Chang Y-H, Lin Y. Targeting STAT1 in both Cancer and insulin resistance diseases. Curr Protein Pept Sci. 2016;18(2):181-8. https://doi.org/10.2174/1389203718666161117114735.

54. Zhao P, Stephens JM. STAT1, NF-KB and ERKs play a role in the induction of lipocalin-2 expression in adipocytes. Mol Metab. 2013;2(3):161-70. https://doi.org/10.1016/j.molmet.2013.04.003.

55. Zhao P, Elks CM, Stephens JM. The induction of Lipocalin-2 protein expression in vivo and in vitro. J Biol Chem. 2014;289(9):5960-9. https:// doi.org/10.1074/jbc.M113.532234.

56. Lu B, Garrido N, Spelbrink JN, Suzuki CK. Tid1 isoforms are mitochondrial DnaJ-like chaperones with unique carboxyl termini that determine cytosolic fate. J Biol Chem. 2006;281(19):13150-8. https://doi.org/10.1074/jbc. M509179200.

57. Yang X, Liu L, Sternberg D, Tang L, Galinsky I, DeAngelo D, et al. The FLT3 internal tandem duplication mutation prevents apoptosis in Interleukin-3-deprived BaF3 cells due to protein kinase a and ribosomal S6 kinase 1-mediated BAD phosphorylation at serine 112. Cancer Res. 2005;65(16):7338-47. https://doi.org/10.1158/0008-5472.can-04-2263.

58. Watanabe N, Okada A, Umezawa M. FLT3-ITD activates RSK1 to enhance proliferation and survival of AML cells by activating MTORC1 and eIF4B cooperatively with PIM or PI3K and by inhibiting bad and BIM. Cancers. 2019;11(12):1827. https://doi.org/10.3390/cancers11121827.

59. Wang Y, Yang J, Hong T, Chen X, Cui L. SIRT2: controversy and multiple roles in disease and physiology. Ageing Res Rev. 2019;55:100961. https:// doi.org/10.1016/j.arr.2019.100961. 
60. Xu L, Wang L, Zhou L, Dorfman RG, Pan Y, Tang D, et al. The SIRT2/CMYC pathway inhibits peroxidation-related apoptosis in Cholangiocarcinoma through metabolic reprogramming. Neoplasia. 2019;21(5):429-41. https://doi.org/10.1016/j.neo.2019.03.002.

61. Kim AY, Bommeljé CC, Lee BE, Yonekawa Y, Choi L, Morris LG, et al. SCCRO (DCUN1D1) is an essential component of the E3 complex for Neddylation*. J Biol Chem. 2008;283(48):33211-20. https://doi.org/10.1074/jbc. M804440200.

62. Huang DT, Ayrault O, Hunt HW, Taherbhoy AM, Duda DM, Scott DC, et al. E2-RING expansion of the NEDD8 cascade confers specificity to cullin modification. Mol Cell. 2009;33(4):483-95. https://doi.org/10.1016/j. molcel.2009.01.011.

\section{Publisher's Note}

Springer Nature remains neutral with regard to jurisdictional claims in published maps and institutional affiliations.

- fast, convenient online submission

- thorough peer review by experienced researchers in your field

- rapid publication on acceptance

- support for research data, including large and complex data types

- gold Open Access which fosters wider collaboration and increased citations

- maximum visibility for your research: over $100 \mathrm{M}$ website views per year

At BMC, research is always in progress.

Learn more biomedcentral.com/submissions 